\title{
Correction to: Sulforaphane protects granulosa cells against oxidative stress via activation of NRF2-ARE pathway
}

\author{
Md. Mahamodul Hasan Sohel ${ }^{1,2,3} \cdot$ Ahmed Amin $^{1,4} \cdot$ Sigit Prastowo $^{1,5} \cdot$ Luis Linares-Otoya $^{1} \cdot$ Michael Hoelker $^{1}$. \\ Karl Schellander ${ }^{1} \cdot$ Dawit Tesfaye $^{1}$
}

Published online: 6 November 2018

(C) Springer-Verlag GmbH Germany, part of Springer Nature 2018

\section{Correction to: Cell and Tissue Research https://doi.org/10.1007/s00441-018-2877-z}

There is an error in the original publication of this paper. Figures 1-6 were shown in the wrong version, thus corrected figures provided below:

The original article has been corrected.

The online version of the original article can be found at https://doi.org/ $10.1007 / \mathrm{s} 00441-018-2877-\mathrm{z}$

Dawit Tesfaye

tesfaye@itw.uni-bonn.de

1 Animal Breeding and Husbandry Group, Institute of Animal Science, University of Bonn, Endenicher Allee 15, 53115 Bonn, Germany

2 Genome and Stem Cell Centre, Erciyes University, 38039 Kayseri, Turkey

3 Department of Animal Science, Faculty of Agriculture, Erciyes University, 38039 Kayseri, Turkey

4 Faculty of Agriculture, Department of Animal Production, Cairo University, Giza, Egypt

5 Department of Animal Science, Sebelas Maret University, Surakarta, Indonesia 


\section{0-50\% confluence \\ 40 \\ $\min$ \\ $24 \mathrm{~h}$}
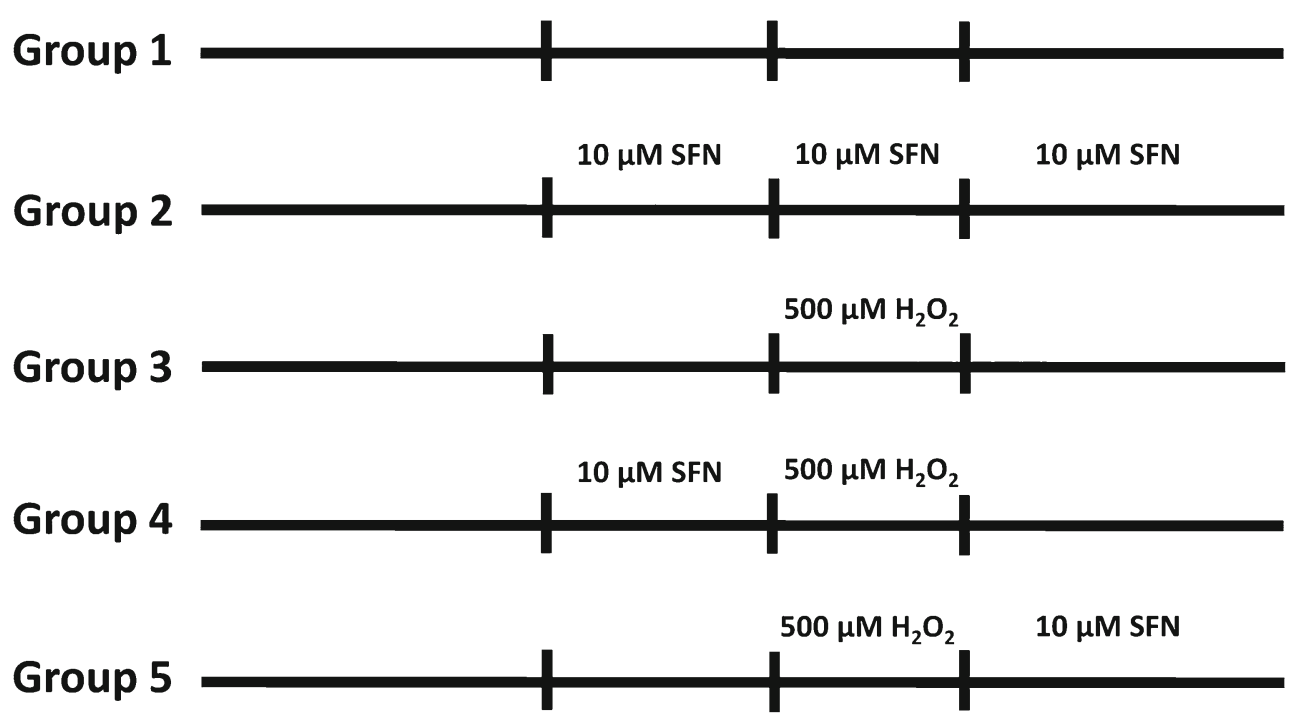

Fig. 1 Simplified experimental groups. Cells in all groups were grown to 40-50\% confluence and continued different treatments under optimum culture conditions. Group 1: no treatment, cells were grown with complete medium; group 2: cells were treated with $10 \mu \mathrm{M} \mathrm{SFN}$; group 3: cells were continued with complete medium up to $40-50 \%$ confluence

followed by an incubation with $500 \mu \mathrm{M} \mathrm{H}_{2} \mathrm{O}_{2}$ for $40 \mathrm{~min}$ and continued with complete medium; group 4: cells were treated with $10 \mu \mathrm{M} \mathrm{SFN}$ for $24 \mathrm{~h}$ followed by 40 -min incubation with $500 \mu \mathrm{M} \mathrm{H}_{2} \mathrm{O}_{2}$; group 5: cells were continued with complete medium followed by an incubation with $10 \mu \mathrm{M} \mathrm{SFN}+500 \mu \mathrm{M} \mathrm{H}_{2} \mathrm{O}_{2}$ for 40 min and continued with $10 \mu \mathrm{M}$ SFN 

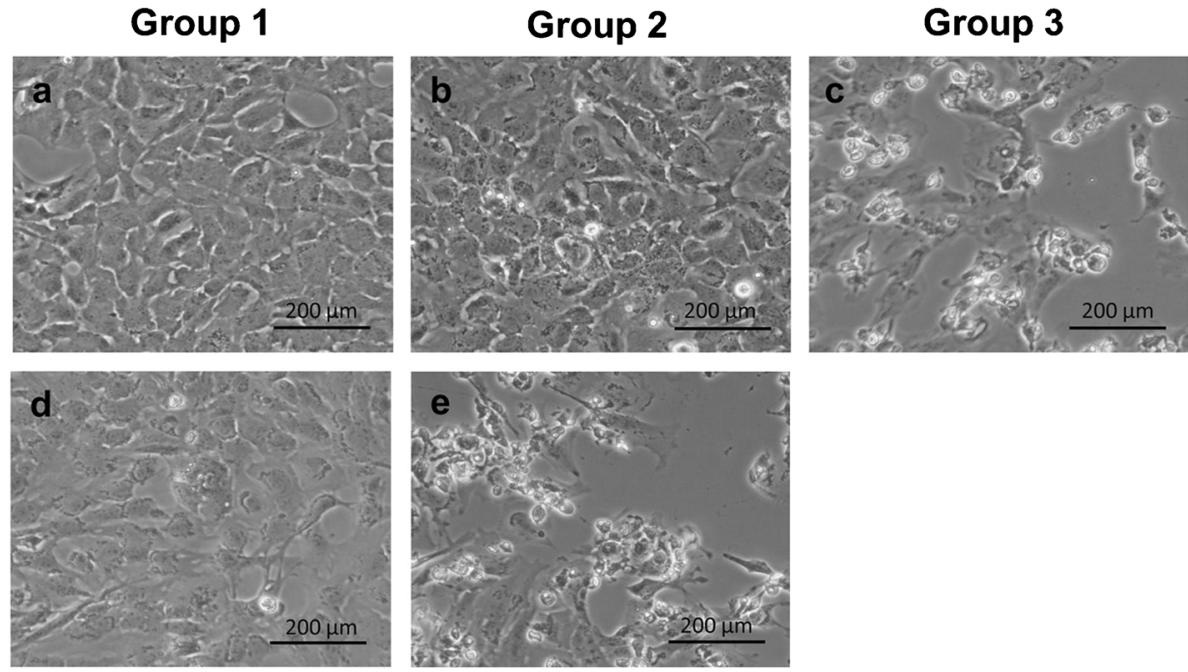

Group 4

\section{Group 5}

$\mathbf{f}$

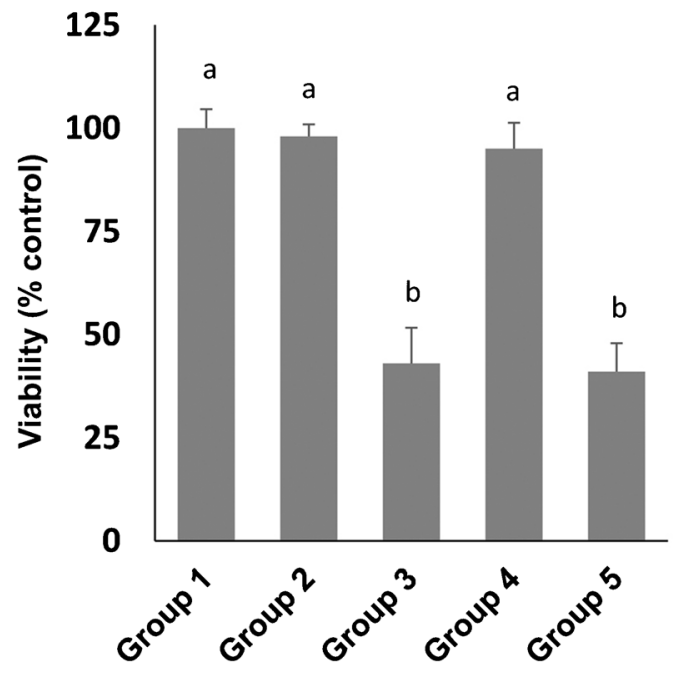

g

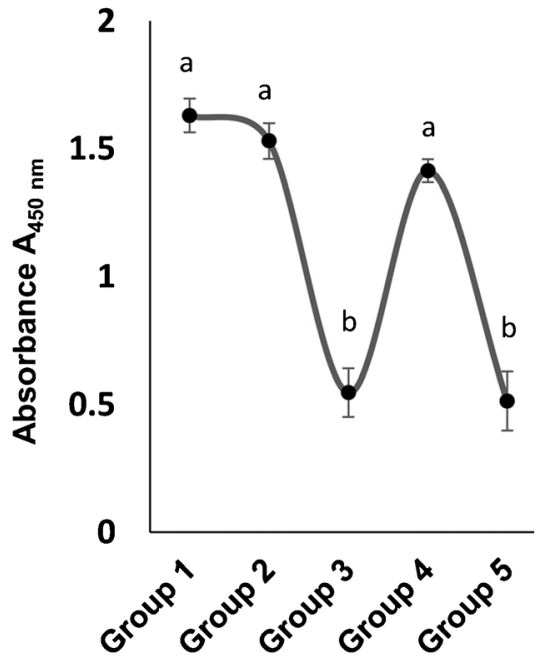

Fig. 2 Effects of different treatments on cellular morphology, viability and cytotoxicity. a-e Representative micrographs of different treatments $(n=3)$, scale bar $200 \mu \mathrm{m}$. f Cells were subjected to trypan blue dye exclusion test and were counted using a hemocytometer for cell viability. g Cytotoxicity of different treatments was determined using
WST-1 kit using an ELISA reader. Data are presented as mean \pm SEM $(n=3)$ of three independent experiments. Different superscript letters $(a, b)$ denote a significant difference between groups, such that groups not sharing a similar letter are significantly different from each other $(\mathrm{p}<0.001)$ 

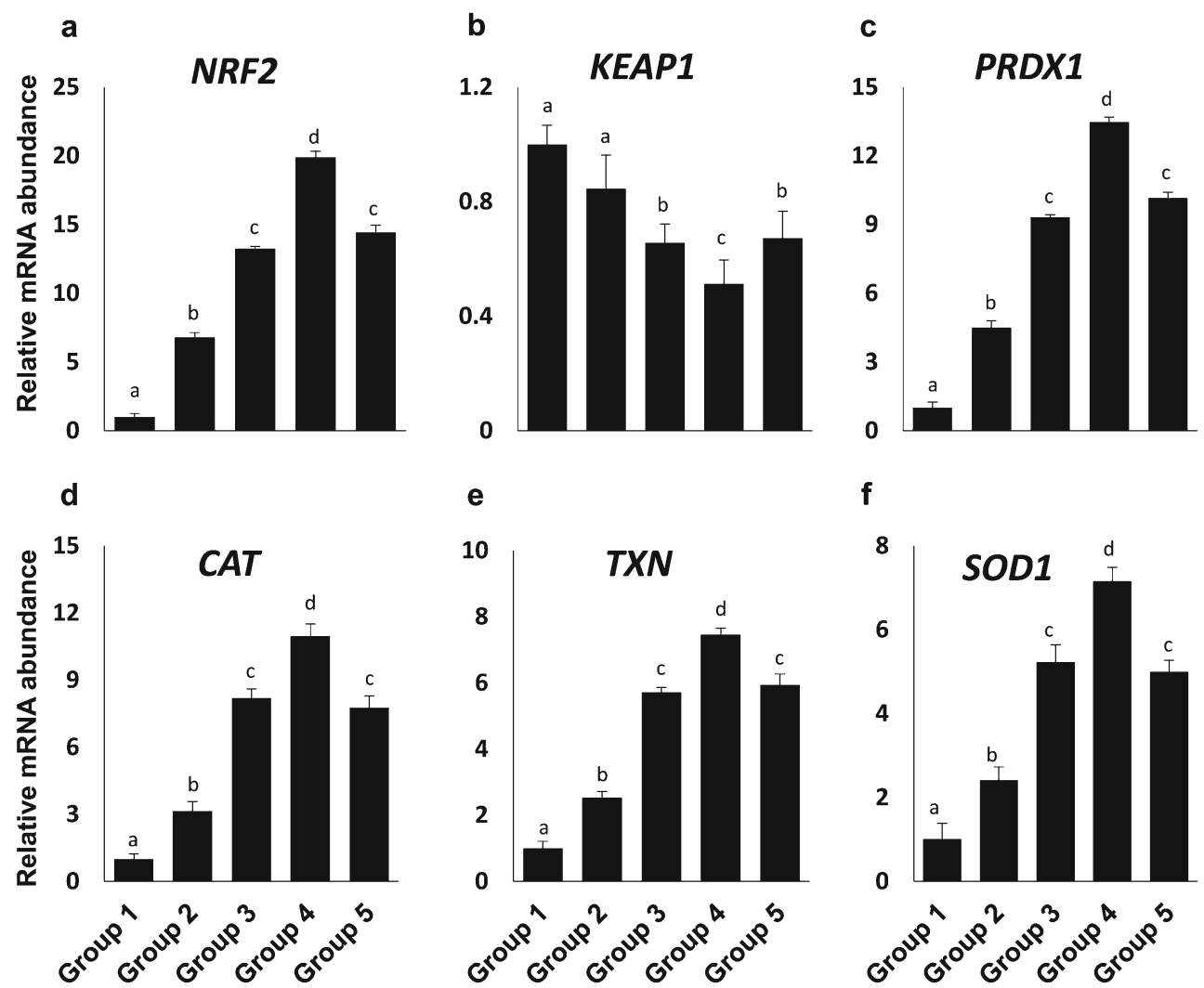

Fig. 3 (a-f) Expression of NRF2, KEAP1 and candidate genes downstream to NRF2 activation. Expression of genes was quantified three biological replicates. Different superscript letters $(a, b, c, d)$ denote a significant difference between groups $(p<0.05)$ as determined by using $\mathrm{qRT}-\mathrm{PCR}$ and relative abundance was analyzed using Student's $t$ test 


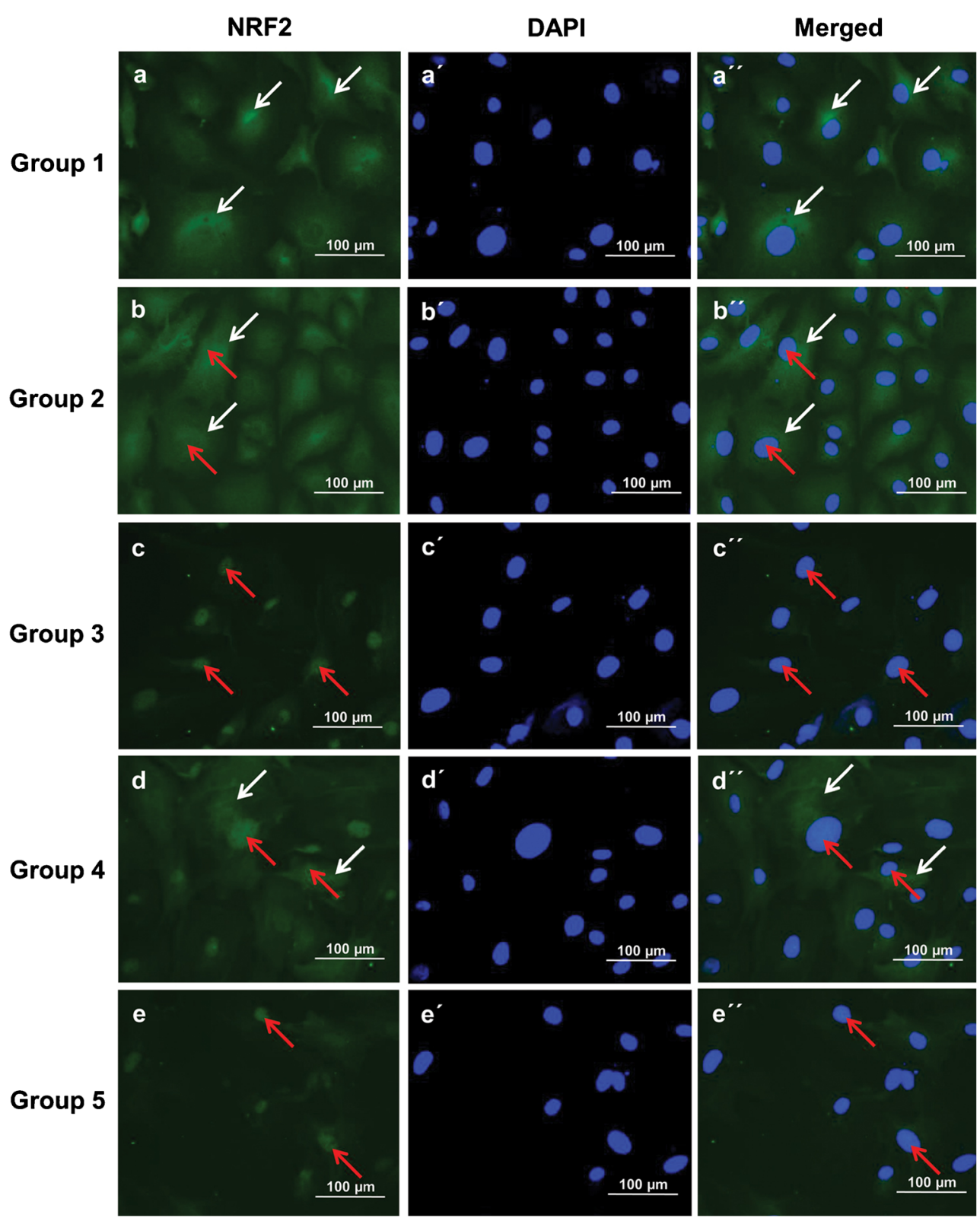

Fig. 4 Nuclear translocation of NRF2. Following different treatments, GCs were subjected to immunocytochemistry to localize NRF2 proteins. Images were acquired with a fluorescence microscope using a green fluorescence filter. Representative images showing the location of
NRF2 proteins in green $(\mathbf{a}-\mathbf{e})$, while nuclear staining with DAPI $\left(\mathbf{a}^{\prime}-\mathbf{e}^{\prime}\right)$ and merged image of NRF2 protein and DAPI (a" $\left.\mathbf{a}^{\prime \prime} \mathbf{e}^{\prime \prime}\right)$. Scale bar 100 $\mu \mathrm{m}$. White arrows indicate NRF2 in the cytoplasm, while red arrows represent the translocated NRF2 in the nucleus 


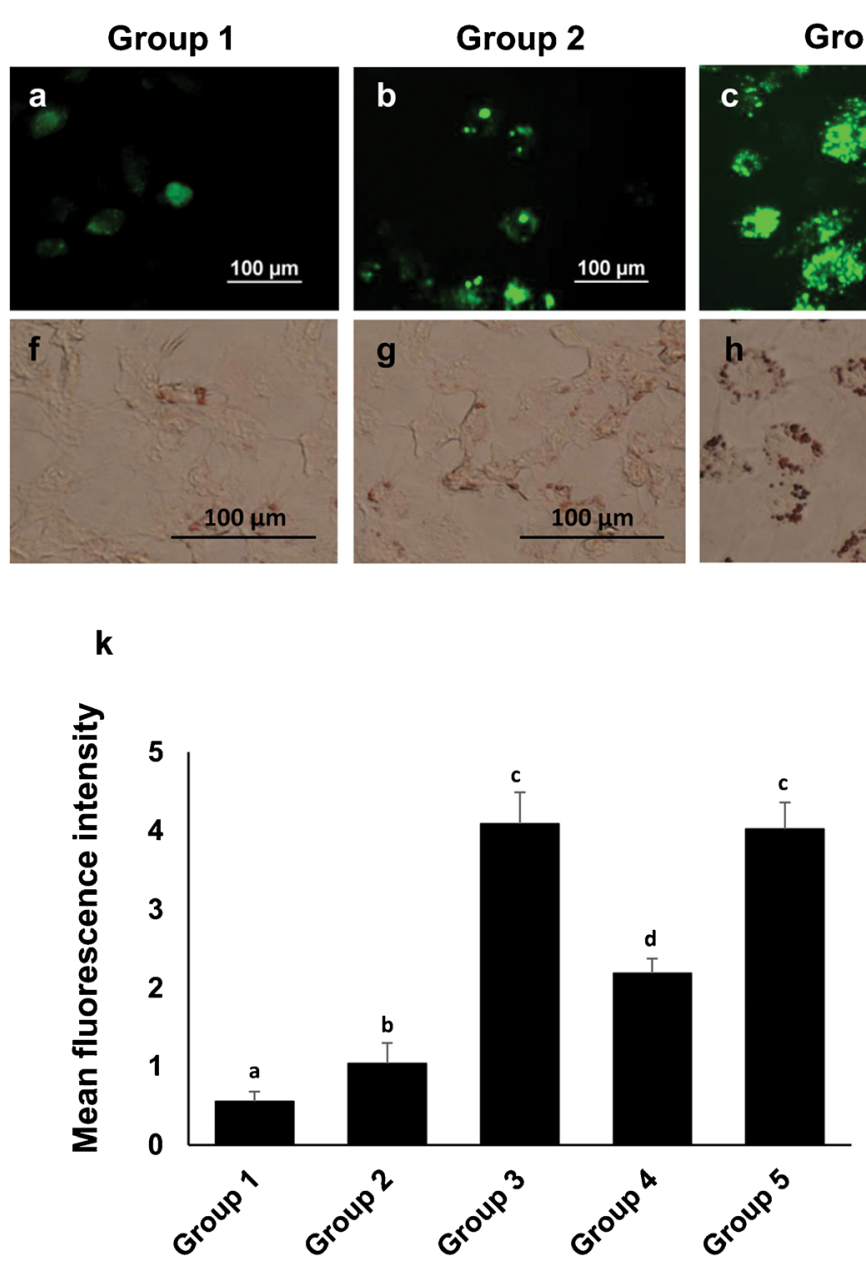

Fig. 5 ROS and lipid droplet accumulation in GCs after different treatments according to Fig. 1. GCs were (a-e) washed and loaded with H2DCFDA (15 $\mu \mathrm{M}$ for $20 \mathrm{~min})$ and visualized under a fluorescent microscope, scale bar $100 \mu \mathrm{m}$. $\mathbf{f}-\mathbf{j}$ Following treatments, GCs were loaded with Oil Red O stain working solution (for $40 \mathrm{~min}$ ), washed several times and visualized with an inverted microscope $(n=3)$. Scale bar $100 \mu \mathrm{m}$. k Mean fluorescence intensity was quantified by ImageJ software from five nonoverlapping fields per well; experiments were performed in triplicate, ${ }^{* * *} p<0.001$. Data are presented as mean $\pm \mathrm{SD}$ 


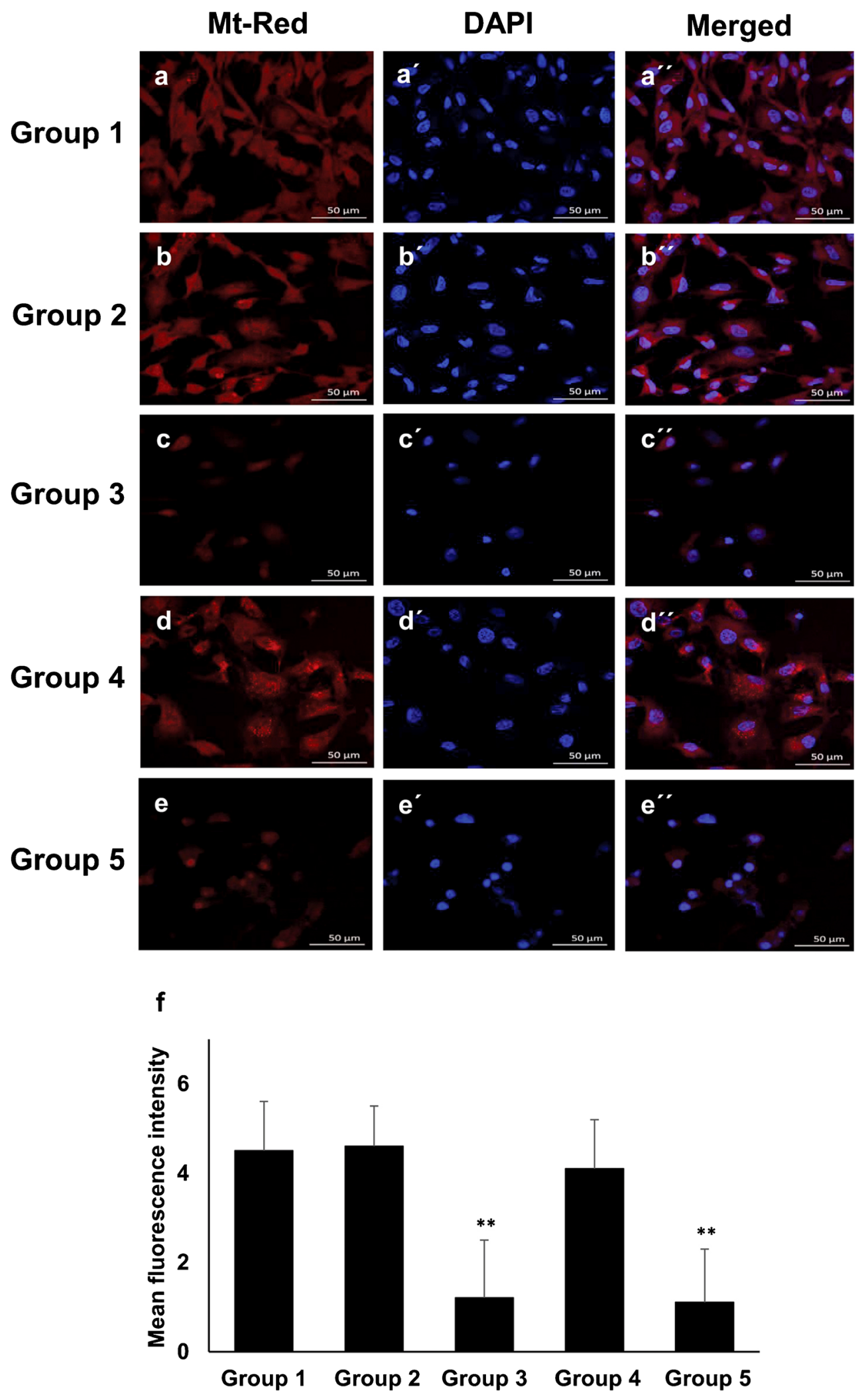

Fig. 6 Mitochondrial activity in GCs of different treatment groups. GCs were treated according to the experimental plan and $100 \mathrm{nM}$ Mito Tracker red dye was added and incubated for $45 \mathrm{~min}$ at $37{ }^{\circ} \mathrm{C}$. Images were active mitochondria in red, while $\mathbf{a}^{\prime}-\mathbf{e}^{\prime}$ show nuclear staining with DAPI in blue and $\mathbf{a}^{\prime \prime}-\mathbf{e}^{\prime \prime}$ show merged image of active mitochondria and nucleus staining. Scale bar $50 \mu \mathrm{m}, n=3$ 